\title{
Montesquieu, CEuvres complètes, t. XIII: Spicilège
}

\section{Domenico Felice}

\section{(2) OpenEdition \\ Journals}

\section{Edizione digitale}

URL: https://journals.openedition.org/studifrancesi/39412

DOI: 10.4000/studifrancesi.39412

ISSN: 2421-5856

\section{Editore}

Rosenberg \& Sellier

\section{Edizione cartacea}

Data di pubblicazione: 1 décembre 2004

Paginazione: 373-374

ISSN: 0039-2944

\section{Notizia bibliografica digitale}

Domenico Felice, «Montesquieu, Eruvres complètes, t. XIII: Spicilège», Studi Francesi [Online], 143 (XLVIII | II) | 2004, online dal 30 novembre 2015, consultato il 19 mai 2021. URL: http:// journals.openedition.org/studifrancesi/39412 ; DOI: https://doi.org/10.4000/studifrancesi.39412

Questo documento è stato generato automaticamente il 19 mai 2021.

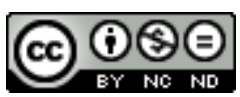

Studi Francesi è distribuita con Licenza Creative Commons Attribuzione - Non commerciale - Non opere derivate 4.0 Internazionale. 


\title{
Montesquieu, CEuvres complètes, $\mathrm{t}$. XIII: Spicilège
}

\author{
Domenico Felice
}

\section{NOTIZIA}

MONTESQUIEU, Cuvres complètes, t. XIII: Spicilège, edité par Rolando MINUTI et annoté par Salvatore ROTTA, Oxford-Napoli, Voltaire Foundation-Istituto Italiano per gli Studi filosofici, 2002, pp. xv-704.

1 Procede, seppure molto a rilento, la nuova edizione critica delle Euvres complètes de Montesquieu, intrapresa da un'équipe internazionale di studiosi aderenti alla Société Montesquieu e stampata dalla Voltaire Foundation e dall'Istituto Italiano per gli Studi Filosofici. Dopo l'uscita del primo tomo (dei quattro previsti) della Correspondance e del tomo II dell'intera collezione (ne sono previsti in tutto 22), dedicato alle Considérations sur les causes de la grandeur des Romains et de leur décadence (1734) e alle Réflexions sur la monachie universelle en Europe (1734), ecco ora la pubblicazione del tomo XIII, contenente lo Spicilège, curato da Rolando Minuti e annotato dal compianto Salvatore Rotta. Dei tre finora venuti alla luce, quest'ultimo tomo è senza dubbio - dal punto di vista di un'edizione che ambisce a definirsi «critica» - il più «riuscito», sia per l'estrema precisione con cui è stata effettuata la nuova collazione integrale sul testo manoscritto sia, soprattutto, per l'ampio, dotto e accuratissimo apparato critico: un tomo che merita, a nostro avviso, di essere assunto a modello da parte degli studiosi impegnati nell'elaborazione delle edizioni dei restanti testi della collezione.

2 Com'è noto, lo Spicilège è una complessa raccolta di pensieri, osservazioni occasionali, estratti di letture, appunti di lavoro, ritagli di giornali, ecc., pubblicato per la prima volta, ma in modo assai parziale, a cavallo tra Ottocento e Novecento, dal giurista Henri Barckhausen nella raccolta, edita dal barone Gastone di Montesquieu, intitolata Pensées et fragments inédits de Montesquieu (2 voll., Bordeaux, Gounouilhou, 1899-1901). Esso consta di due parti (entrambe finemente analizzate da Salvatore Rotta nella sua 
«Introduction»): la prima, costituita dal cosiddetto «Recueil Desmolets», risale agli anni tra il 1703, o il 1704, e la prima metà del 1705. Di autore ignoto, tale «Recueil» fu donato verso la fine del 1713 a Montesquieu, che ne ricopiò gran parte delle «note» (con nuovi titoli e l'aggiunta di riflessioni personali) nel suo Spicilège, dal colto prete oratoriano Pierre-Nicolas Desmolets, conosciuto dal Président fin dagli anni (1700-1705) dei suoi studi classici presso il collegio di Juilly, vicino Parigi. Benché nel complesso «sans grande valeur» (p. 23), il «Recueil» in questione rivela nondimeno alcuni precisi centri di interesse del suo anonimo compilatore (quali, in particolare, la storia sacra e profana, i costumi dei Greci e dei Romani e l'attualità scientifica) e consente di abbozzarne, secondo Rotta, il seguente «ritratto»: «Il s'agit probablement d'un jeune homme qui, pendant deux ou trois années de sa vie [...] a fixé sur le papier pour luimême ce qui l'a frappé dans des lectures versatiles, décousues, quelquefois cursives, de journaux et de livres. Il n'avait pas de projet précis; en tout cas, il ne l'a pas révélé [...]. Cette abeille butineuse fait ce que le titre \{Spicilège\} annonce: il glane, il picore. Il montre dans le choix de ses thèmes une curiosité dispersée, mais aussi de la verve. Il hasarde de temps en temps des hypothèses, souvent très mal fondées, quelquefois téméraires, ce qui peut le faire soupçonner de libertinage. Mais si on regarde le fond de sa pensée, jusque dans ses moments d'audace, on découvre le traditionaliste qui rentre toujours dans les rangs de l'Église et veut que la raison soit soumise aux mystères de la foi. Il a toutefois des aversions marquées, fort significatives: il déteste l'Inquisition et la censure des livres; il flétrit les tyrans; il ne croit pas aux possessions diaboliques [...]; il n'aime pas l'évangélisation à fil de l'épée; il préfère les écoles aux couvents [...]; il fait des réserves sur les miracles. Bref, on peut le mettre au nombre des catholiques éclairés, tels que Desmolets et - pourquoi pas? - Montesquieu» (p. 24).

La seconda parte, assai più ampia (quasi il triplo delle «note» trascelte dal «Recueil Desmolets»), è il vero e proprio Spicilège di Montesquieu, la cui stesura si protrasse, con varie interruzioni, dal 1715 al 1753. Tra le novità più rilevanti introdotte dal Président vanno segnalati in particolare: lo spostamento dell'attenzione dai giornali scientifici (segnatamente, il «Journal des savants» e i «Mémoires de Trévoux») a quelli politici, come la «Gazette d'Amsterdam», la «Gazette d'Utrecht», la «Gazette de France», il «Craftsman», ecc; la «registrazione» di notizie anche orali o di conversazioni tra Montesquieu ed eminenti uomini di cultura del suo tempo (dal gesuita Jean-François Fouquet, inesauribile fonte di informazioni sulla Cina, dove egli aveva soggiornato per oltre vent'anni, al duca di Saint-Simon, passando per Fontenelle, Fréret o l'italiano Gaspare Cerati); la presenza, infine, di numerose citazioni di passi controversi dell'Antico Testamento. Queste, ed altre innovazioni di minor rilievo, rivelano senza dubbio, da parte di Montesquieu, un campo di curiosità assai più vasto e diversificato rispetto a quello dell'anonimo autore del «Recueil», anche se bisogna decisamente rinunciare «à l'illusion de trouver dans le Spicilège l'esquisse des grands ouvrages» del Président (p. 34). Vi si rinvengono piuttosto le tracce di suoi scritti rimasti allo stato di progetto, come ad esempio la storia del regno di Luigi XIV. Si deve pertanto -conclude Rotta - prendere lo Spicilège per quello che effettivamente è: «une suite décousue de réflexions [...] et un vaste dépôt de nouvelles et d'anecdotes». Diversamente da quel che riteneva André Masson (curatore, nel 1944, della prima importante, sebbene ancora incompleta, edizione dell'opera), sono i brani o i ritagli di giornali, presenti in gran quantità in questo «difficile» testo, «[sa] partie la plus cohérente», quella che meglio «si lega» ad alcuni temi dominanti del pensiero di Montesquieu, quali il problema demografico, l'andamento dei cambi, la situazione finanziaria dell'Inghilterra del 
tempo o quella politica delle ormai decrepite repubbliche aristocratiche italiane (Venezia, Genova, ecc.). 0 ancora: la persistenza del dispotismo in Russia, la condizione sempre più disastrosa dell'impero persiano dei Safàwidi, le difficoltà della penetrazione inglese nell'America spagnola o in India, le minacce russe contro l'indipendenza della Polonia, l'apertura di nuove rotte nel commercio internazionale, ecc.; tutti temi da cui emerge un Montesquieu assiduo e acuto osservatore dell'attualità politica e geopolitica europea ed extraeuropea del suo tempo (pp. 34-35). Cosicché, seppure largamente «inferiore», per ricchezza e profondità, alle Mes Pensées, anche questo «carnet de travail» - le cui singole «note» restano peraltro in gran parte di difficile datazione - ci consente di comprendere meglio e di penetrare più a fondo nel laboratorio intellettuale di un pensatore «aussi profond que malicieux» (p. 36), qual è il Président. 